“(C) 2021 IEEE. Personal use of this material is permitted. Permission from IEEE must be obtained for all other uses, in any current or future media, including reprinting/republishing this material for advertising or promotional purposes, creating new collective works, for resale or redistribution to servers or lists, or reuse of any copyrighted component of this work in other works." 


\title{
An Elliptical Cylindrical Shaped Transmitarray for Wide-Angle Multibeam Applications
}

\author{
Li-Zhao Song, Pei-Yuan Qin, Shu-Lin Chen, and Y. Jay Guo
}

\begin{abstract}
A transmitarray antenna with an elliptical cylindrical shape is presented for a wide-angle multibeam radiation in this paper. The transmitarray has a cylindrical radiating aperture with an elliptical cross section, namely, elliptical cylindrical shape. Multiple feeds can be placed on the middle horizontal plane to realize multiple beams. Inspired by a twodimensional (2-D) Ruze lens, the antenna shape and the phase compensation are jointly designed according to the desired maximal beam direction. Innovative methods including a feed refocusing analysis and a virtual focal length are utilized to achieve the phase compensation across the threedimensional (3-D) aperture for multiple beam radiations with a small scanning loss. In order to validate the proposed antenna, a prototype operating in the millimeter-wave band has been designed, fabricated and measured. By changing the position of the feeding gain horn along the refocusing arc, the main beam of antenna can be scanned to eleven directions. The measured peak boresight realized gain is $27 \mathrm{dBi}$ at $70.5 \mathrm{GHz}$ and a beam coverage of $\pm 43^{\circ}$ with a less than $2.7-\mathrm{dB}$ scanning loss is obtained.
\end{abstract}

Index Terms - Multibeam antenna, millimeter wave, transmitarray, wide angle coverage.

\section{INTRODUCTION}

Millimeter-wave (mm-wave) multibeam antennas are widely hailed as a key antenna technology for the fifth generation $(5 \mathrm{G})$ and beyond wireless communication networks, supporting high data rate communications with an increased spectral efficiency [1]. By leveraging the much smaller wavelength at mm-wave bands as compared to that in the sub-6-GHz frequencies, antenna array with a larger electrical aperture size can be deployed into the same physical area, leading to a much higher array directivity and gain. This will compensate for the path loss at mm-wave frequencies. Meanwhile, multibeam antennas can provide a wide beam coverage to serve a number of distributed users. To this end, mm-wave multibeam antennas have attracted considerable attention from both academia and industry. Compared with the existing 5G digital/hybrid beamforming technologies [2-3], passive or analogue multibeam antennas offer several significant commercial advantages, including lower manufacturing costs, reduced power consumptions and more deployment flexibilities. The existing passive multibeam antennas include those based on lens [4-6], transmitarrays [7-9], reflectarrays [10-12] and beamforming circuits, e.g., Butler-matrix-based antennas [13-14]. Among them, transmitarrays/reflectarrays have their own merits of achieving high gains without needing lossy transmissionline-based feeding networks. Furthermore, compared with reflectarrays, transmitarrays do not have the issue of feed blockage as its feed and the main beam are located on different sides of the aperture [15-20]. Motivated by these advantages, substantial research efforts have been devoted to multibeam transmitarrays.

One popular technology is to utilize the bifocal phase compensation method initially presented in reflectarrays [21]. In [22], circularly-polarized multibeam transmitarrays are developed at 26 $\mathrm{GHz}$, generating five pencil beams that cover a range of $\pm 33^{\circ}$ with a scanning loss of $1.2 \mathrm{~dB}$. The peak boresight gain is $22.2 \mathrm{dBic}$. In [23], a seven-beam transparent transmitarray at $28.5 \mathrm{GHz}$ is presented. It has a peak boresight gain of $25 \mathrm{dBi}$ covering a range of $\pm 30^{\circ}$ with a scanning loss of $3.5 \mathrm{~dB}$. Another technology is to develop metamaterial-based planar multibeam transmitarrays. A multibeam transmitarray using Jerusalem cross elements is demonstrated at 28 $\mathrm{GHz}$ band, achieving an angular coverage of $\pm 27^{\circ}$ with a scanning loss of $3.7 \mathrm{~dB}$ [24]. The maximum gain is $24.2 \mathrm{dBi}$. A multibeam folded transmitarray is demonstrated in [25] with five beams covering up to $\pm 10^{\circ}$ at $30 \mathrm{GHz}$. Besides, by using a sliding transmitting aperture or feed, circularly-polarized mechanical beam switching transmitarrays are demonstrated at Ka band [26-27]. An asymmetric beam scanning range of $0^{\circ}$ to $50^{\circ}$ is achieved for satellite-on-the-move (SOTM) ground terminal applications. The beam switching methods could be employed to achieve multibeam radiations when multiple feeds are implemented. In [28], a linear multibeam transmitarray is designed based on a sliding aperture concept at $5.8 \mathrm{GHz}$, where eight beams are produced with a 3 -dB beam coverage of $\pm 42^{\circ}$. Since it is a linear array, a medium gain up to $13.9 \mathrm{dBi}$ is achieved.

In light of the above discussion, it is noticed that most of the published high-gain mm-wave multibeam transmitarrays have a beam coverage of about $60^{\circ}\left( \pm 30^{\circ}\right)$. This would limit the applications of the technology. It is of practical importance to industry that the beam coverage is further extended in order to cover a larger angular range to enable point-to-multi-point communications. Furthermore, it is desirable to have multibeam transmitarrays operating at higher frequency bands. The mm-wave band above $50 \mathrm{GHz}$ offers a large spectrum, and it has a great potential to support future high speed wireless networks. Moreover, the abovementioned transmitarrays are all planar ones. The technologies for shaped multibeam transmitarrays are still lacking. A curved cylindrical transmitarray is demonstrated in [29], providing a simulated beam scanning range of $82^{\circ}$ with a $3.9 \mathrm{~dB}$ scanning loss. Another cylindrical transmitarray [30] achieves a mechanical beam scanning range of $\pm 15^{\circ}$ using a superposition method. More specifically, its transmitting aperture is divided into six sectors with different beam directions. The feed horn is rotated to illuminate two sectors at a time, thereby pointing the main beam of the array to a specific direction. Unfortunately, it cannot realize multibeam radiation as its feed horn is rotated at a fixed position.

In this paper, inspired by the two-dimensional (2-D) wide-angle scanning metal-plate lenses introduced by Ruze [31], an elliptical cylindrical transmitting aperture is developed for the first time for multibeam applications. The proposed transmitarray utilizes the shape of the aperture as an additional degree of freedom. The aperture shape and the phase compensation are jointly designed according to the desired maximum beam direction, leading to different focal lengths for different beam directions. To validate the concept of the proposed multibeam system, a prototype is designed with a standard feed horn placed at different positions to radiate the corresponding beams. The transmitarray can realize a measured peak boresight gain of $27 \mathrm{dBi}$ at $70.5 \mathrm{GHz}$ and a beam coverage up to $\pm 43^{\circ}$ along the horizontal plane with a 2.7-dB scanning loss. Compared with many published multibeam transmitarrays, the proposed array has a larger beam scanning range with a small scanning loss. Furthermore, the developed array is one of the very few transmitarrays that operate at higher mm-wave frequencies with a curved shape. 
The rest of this paper is organized as follows. In Section II, detailed design methods for multibeam transmitarray are introduced. These developed methods are verified through simulations in Section III. In Section IV, a multibeam transmitarray prototype is fabricated and measured, and the simulated and measured results are compared. The paper concludes in Section V.

\section{Multibeam TransmitarRay Design}

\section{A. Transmitarray Shape and Phase Calculation}

According to the 2-D Ruze lens theory [31], the phase compensation along the transmitarray aperture is calculated based on the predefined largest beam angles. As illustrated in Fig. 1, we assume that $\mathrm{O}_{1}$ and $\mathrm{O}_{2}$ are the focal points for two symmetrical radiation beams in xoy plane. These two beams are respectively denoted as beam 1 and beam 2 at $\pm \alpha$ angles, where $\alpha\left(0<\alpha<90^{\circ}\right)$ is the maximum beam angle measured from $+x$-axis. They are equal to the feed offset angles. $l_{1}$ and $l_{3}$ represent the distance from the points $\mathrm{O}_{1}$ and $\mathrm{O}_{3}$ on the focal arc to a random element $(x, y)$ on the aperture, respectively. $l_{0}$ is the focal length of $\mathrm{O}_{1}$.

Referred to [31], for beam 1 radiation at $\alpha$ angle, the element phase compensation must satisfy:

$k_{0} l_{1}-\varphi_{e}(x, y)=k_{0}\left(l_{0}+x \cos \alpha+y \sin \alpha\right)-\varphi_{e}(0,0)$

$k_{0}$ is the propagation constant in free space. $\varphi_{e}(x, y)$ is the element phase compensation value at $(x, y) . \varphi_{e}(0,0)$ is the element phase compensation at point $(0,0)$. We can calculate $l_{1}$ with:

$l_{1}=\sqrt{\left(x+l_{0} \cos \alpha\right)^{2}+\left(y+l_{0} \sin \alpha\right)^{2}}$

By substituting (2) in (1), and define $u$ as:

$u=\frac{\Delta \varphi_{e}}{k_{0}}=\frac{\varphi_{e}(x, y)-\varphi_{e}(0,0)}{k_{0}}$

one can obtain:

$x^{2} \sin ^{2} \alpha+y^{2} \cos ^{2} \alpha-2 x y \sin \alpha \cos \alpha$

$=u^{2}+2 u l_{0}+2 u y \sin \alpha+2 u x \cos \alpha$

Applying the above procedures for beam 2 radiation at $-\alpha$ angle, another equation will be produced as:

$x^{2} \sin ^{2} \alpha+y^{2} \cos ^{2} \alpha+2 x y \sin \alpha \cos \alpha$

$=u^{2}+2 u l_{0}-2 u y \sin \alpha+2 u x \cos \alpha$

Combining (4) and (5), the ideal transmitarray contour in xoy plane can be achieved as (6), and the phase compensation equation along that is given in (7):

$\left(\frac{x}{l_{0} \cos \alpha}+1\right)^{2}+\left(\frac{y}{l_{0}}\right)^{2}=1$

$\Delta \varphi_{e}=-k_{0} x \cos \alpha$

$\Delta \varphi_{e}$ represents the relative phase compensation at $(x, y)$ with respect to point $(0,0)$. As can be seen from $(6)$, along the multibeam radiation coordinate (xoy plane), the transmitarray shows an elliptical cross section. While in the vertical plane (xoz plane), the transmitarray has a straight contour.

For a multibeam radiation with multiple feeds, initially we assume that the feeds would be placed along the focal arc, as shown in Fig. 1, with the radius of $l_{0}$ and the pivot at $(0,0)$ point. Since the phase compensation along the transmitarray is calculated from the maximal radiation angles at $\pm \alpha$ rather than $0^{\circ}$, there is a phase error $\delta$ for the feed located at the center of the focal arc $\mathrm{O}_{3}$, which generates beam pointing towards $0^{\circ}$ (beam3). This phase error can be derived from (8), where $\Delta \varphi_{e}$ represents the required relative phase compensation value for beam 3 with feed horn at $\mathrm{O}_{3}$.

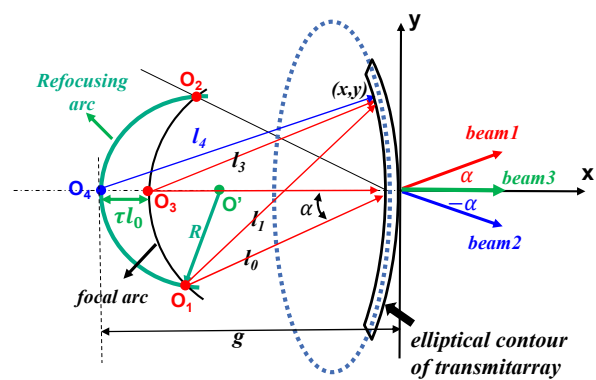

Fig. 1. Horizontal plane (xoy) of transmitarray.

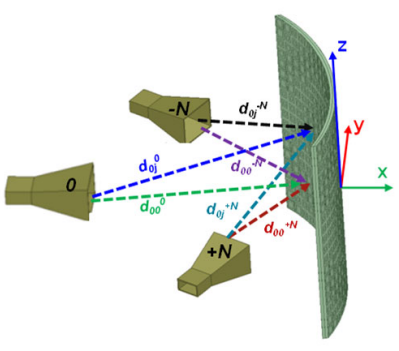

(a)

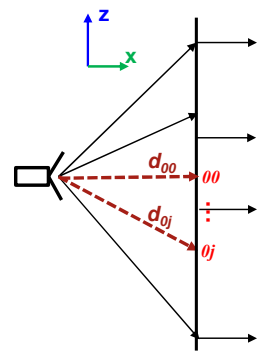

(b)
Fig. 2. Transmitarray configuration with multiple feeds. (a) 3-D schematic. (b) Cross section in xoz plane.

$\delta=\Delta \varphi_{e 3}-\Delta \varphi_{e}$

$=k_{0}\left(\sqrt{\left(x+l_{0}\right)^{2}+y^{2}}-(1-\cos \alpha) x-l_{0}\right)$

To eliminate this phase error, we moved the feed point $\mathrm{O}_{3}$ away from the transmitarray aperture to $\mathrm{O}_{4}$ by $\tau l_{0}$ as illustrated in Fig. 1 . This movement will introduce a phase correction value $\sigma$ which can be calculated from (9), where $\Delta \varphi_{e 4}$ represents the required relative phase compensation value for beam 3 with feed horn at $\mathrm{O}_{4}$.

$\sigma=\Delta \varphi_{e 4}-\Delta \varphi_{e 3}$

$=k_{0}\left(\sqrt{\left(x+(1+\tau) l_{0}\right)^{2}+y^{2}}-\sqrt{\left(x+l_{0}\right)^{2}+y^{2}}-\tau l_{0}\right)$

We can eliminate the phase aberration for beam 3 as long as $\sigma=-\delta$. By doing Taylor series expansions for the variant $y$ of (8) and (9) at $(0,0)$ point and omitting the higher-order terms, the value of the parameter $\tau$ is derived and given in (10).

$\tau=\tan ^{2} \alpha$

Then we would achieve a new focal arc, marked as the refocusing arc in Fig. 1, and the pivot moves from $(0,0)$ to $\mathrm{O}^{\prime}(R-g, 0)$, where $g$ is the new focal length at $\mathrm{O}_{4}$ with the value of $(1+\tau) l_{0}$, and $R$ can be calculated from cosine law as:

$R=\frac{2 g l_{0} \cos \alpha-l_{0}{ }^{2}-g^{2}}{2 l_{0} \cos \alpha-2 g}$

When the feed source is moved along the refocusing arc, the beam of the transmitarray can be directed to different angles.

\section{B. Phase Calculation along xoz Plane}

Since this multibeam transmitarray shows an elliptical cylindrical shape, the phase compensation calculation is divided into two parts, i.e., along xoy plane (elliptical arc in horizontal plane) and along xoz plane (vertical straight plane). We number the elements along xoy and xoz coordinates as $i$ and $j$ respectively, as shown in Fig. 2, where multiple feed horns, numbered as $-N$ to $+N$, are used in the $\mathrm{z}=0$ plane. The distance between each horn and the element at $(0,0)$ is labelled as $d_{00^{-N}}$ to $d_{00}{ }^{+N}$. The distance between each horn and the element along xoz plane is labelled as $d_{0 j}{ }^{-N}$ to $d_{0 j}{ }^{+N}$. In Section II.A, the phase 
distribution along xoy plane is investigated, where feeds at different positions would radiate beams to different angles. On the other hand, along xoz plane, boresight beams are radiated for all the different feeds. The phase distribution along this plane will be studied in this sub-section.

For the boresight radiation on xoz plane, taking the zero column (central column along $\mathrm{z}$ axis) as an example, the phase compensation along $\mathrm{z}$ axis, as illustrated in Fig. 2(b), can be calculated with:

$\Delta \varphi_{s}=k_{0}\left(d_{0 j}-d_{00}\right)$

which is related to the focal length $d_{00}$.

As shown in Fig. 2(a), after refocusing, the focal length of horn 0 $\left(d 00^{\circ}\right)$ is different from that of the horns $-\mathrm{N}\left(d 00^{-N}\right)$ and $+\mathrm{N}\left(d 00^{+N}\right)$. As a result, for each gain horn, the phase distribution along $\mathrm{z}$ axis would be different. Therefore, a virtual value of $d_{00}$ needs to be decided according to the system requirements. For example, if the goal is to maximize the gain of the boresight beam, then the $d_{00}$ needs to be close to $d 00^{\circ}$. On the other hand, if the goal is to minimize the gain difference between the boresight beam and other beams, then $d_{00}$ should be about the average value of $d 00^{\circ}$ and $d 00^{+N}$.

For other straight columns parallel with $\mathrm{z}$ axis, the selected $d_{i 0}$ may be different from the central column $d_{00}$. Ideally, $d_{i 0}$ should be calculated for each column according to the system requirements as discussed above. For simplicity, the value of $d_{00}$ for the central column is used as $d_{i 0}$ for other columns.

\section{CONCEPT Verification Through Simulation}

\section{A. Unit Cell}

In this paper, the unit cell employed for the transmitarray is a triplelayer structure with three identical square ring slots printed on two substrates [30], as shown in the inset of Fig. 3. The substrate has a dielectric constant $\varepsilon_{\mathrm{r}}=2.2$ and loss tangent of 0.0009 . Since the transmitarray has an elliptical cylindrical shape, the easiest and most cost-effective way is to bend a planar aperture onto a 3-D-printed frame. This method has been validated in the conformal transmitarrays [32]. As analyzed in [30], there is always a trade-off between the element thickness and the phase range. Therefore, in this work, the overall thickness $h$ is chosen as $0.508 \mathrm{~mm}\left(0.12 \lambda_{0}\right.$ at $\left.72 \mathrm{GHz}\right)$. The element period is $P=2 \mathrm{~mm}$, and the optimized slot width is $w=0.3 \mathrm{~mm}$. By simulating the element with the master-slave boundary and Floquet ports in ANSYS HFSS, its amplitude and phase performance versus $L$ are achieved under a normal incidence wave in Fig. 3. A $340^{\circ}$ phase variation range is realized with a maximum $3-\mathrm{dB}$ transmission loss by tuning slot length $L$ from $1.2 \mathrm{~mm}$ to $1.88 \mathrm{~mm}$ at $72 \mathrm{GHz}$. For the oblique incidence waves, the performance is found to be acceptable [30]. It is not repeated in this paper due to the space limit.

\section{B. Transmitarray Construction and Refocusing for Boresight} Radiation

A multibeam transmitarray with an elliptical cylindrical shape is designed with maximal beam radiation angles of $\pm 45^{\circ}$, and the focal length $l_{0}$ is chosen to be $30 \mathrm{~mm}$ at $\mathrm{O}_{1}$ and $\mathrm{O}_{2}$ by considering the aperture size and the radiation properties of the feed horn. Then, by using (6), the contour along xoy plane can be obtained as (13):

$\left(\frac{x}{15 \sqrt{2}}+1\right)^{2}+\left(\frac{y}{30}\right)^{2}=1$

The phase compensation along this contour is:

$\Delta \varphi_{e}=-\frac{2 \pi}{\lambda_{0}} \cdot \frac{\sqrt{2}}{2} x$

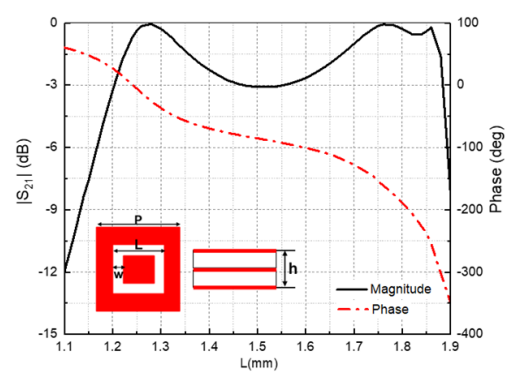

Fig. 3. Simulated element performance at $72 \mathrm{GHz}$.

To precisely arrange elements on this transmitarray and calculate the phase compensation value for each element, we need to make the elliptical arc length as the integral multiples of the element period $P$ and to specify the element positions along the arc. If the $x$ and $y$ in (13) are expressed as:

$$
\left\{\begin{array}{c}
x=15 \sqrt{2}(\cos \varphi-1) \\
y=30 \sin \varphi
\end{array}\right.
$$

we can connect the element position to the related arc length with (16).

$l=\int_{0}^{\varphi} \sqrt{(15 \sqrt{2})^{2} \sin ^{2} \phi+(30)^{2} \cos ^{2} \phi} d \phi$

For example, for the $i$-th element with the arc length $l=i P$ along the xoy plane, the related phase compensation value $\Delta \varphi_{e}$ can be achieved by combining (14)-(16).

To eliminate the phase error for boresight radiation, a refocusing method is adopted as illustrated in Fig. 1. In this design, the feed position $\mathrm{O}_{4}$ can be obtained from (10) as $\tau=1$, resulting in $g=2 l_{0}=60$ $\mathrm{mm}$. For multibeam realizations, different feed horns need to be arranged along the refocusing arc as marked in Fig. 1.

\section{Phase Distribution on 3-D Transmitting Aperture}

As mentioned before, the phase distribution calculation for the whole structure is divided into two parts. For the elliptical arc along xoy plane, (14) is used for the phase calculation. To achieve the phase compensation along the vertical xoz plane, as introduced in Section II.B, a virtual focal length $d_{00}$ needs to be decided for desired radiation performance. In this design, an elliptical cylindrical transmitarray with $25 \times 23=575$ cells is designed using the element demonstrated in Section III.A at $72 \mathrm{GHz}$. A standard gain horn LB-12-15-A from AINFO is placed at each focal point as the feed source, which operates from 60 to $90 \mathrm{GHz}$. Its gain is $15.35 \mathrm{dBi}$ at $72 \mathrm{GHz}$ as noted from the datasheet [33]. The size of the aperture projection on yoz plane is 46.4 $\mathrm{mm} \times 46 \mathrm{~mm}=10.9 \lambda_{0} \times 10.8 \lambda_{0}$ (at $70.5 \mathrm{GHz}$ ), which is used to calculate the aperture efficiency. The dimensions were chosen to make the aperture edge illumination about $-10 \mathrm{~dB}$ when the horn is located at points $\mathrm{O}_{1}$ and $\mathrm{O}_{2}$.

The feed positions for the boresight radiation and $-45^{\circ}$ radiation are $(\mathrm{x}, \mathrm{y})=(-60 \mathrm{~mm}, 0 \mathrm{~mm})$ and $(-21.2 \mathrm{~mm}, 21.2 \mathrm{~mm})$, respectively. In this case, $d 00^{\circ}=g=60 \mathrm{~mm}$ and $d 00^{-N}=30 \mathrm{~mm}$. As illustrated in Section II.B, to balance the radiation performance at $0^{\circ}$ and $\pm 45^{\circ}$, a virtual focal length $d_{00}=36 \mathrm{~mm}$ is chosen to calculate the phase compensation along xoz plane with (12). This virtual focal length is chosen by optimizing the scanning loss between $0^{\circ}$ and $\pm 45^{\circ}$ with a target of less than $3 \mathrm{~dB}$. After combining the vertical phase compensation with the elliptical one from (14), the phase for the whole transmitting aperture is obtained. Then, we simulated the antenna's boresight radiation patterns in xoy (H-plane) and xoz (E-plane) planes as given in Fig. 4(a). It can be noticed that the 3-dB beamwidth in Eplane is wider than that of the H-plane. This is because the chosen 


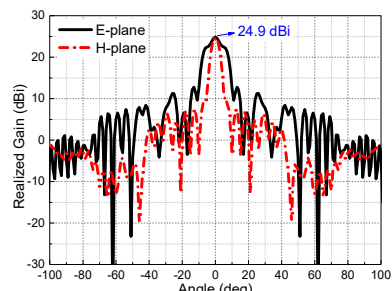

(a)

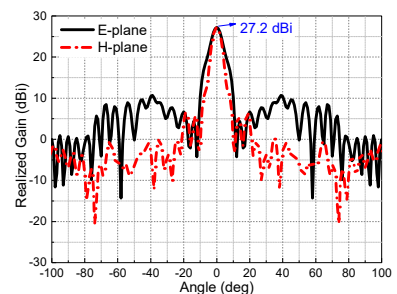

(b)
Fig. 4. Simulated radiation patterns. (a) Boresight radiation patterns with $d_{00}{ }^{0}=60 \mathrm{~mm}$. (b) Boresight radiation patterns with $d_{00}{ }^{0}=48 \mathrm{~mm}$.

virtual focal length $d_{00}=36 \mathrm{~mm}$ for the phase calculation in vertical plane deviates a lot from the focal length $\left(d_{00} 0^{\circ}=60 \mathrm{~mm}\right)$ for $0^{\circ}$ radiation in the elliptical arc plane. Therefore, the refocusing position $(-g, 0)$ of horn 0 for boresight radiation, needs to be optimized to balance the E- and H-plane radiation performance. By changing $g$ ( $g=$ $d 00^{\circ}$ ) to $48 \mathrm{~mm}$, which means moving the gain horn for boresight radiation towards the aperture, the radiation pattern along E-plane is improved as shown in Fig. 4(b), increasing the peak gain from 24.9 $\mathrm{dBi}$ to $27.2 \mathrm{dBi}$. The gain horn positions are unchanged for $\pm 45^{\circ}$ beam directions. In this case, the final refocusing position for horn 0 is decided as $(-g, 0)=(-48 \mathrm{~mm}, 0)$, which is referred as $\mathrm{O}_{4}{ }^{\prime}$ in Fig. 5 .

\section{Feed System for Multibeam Realization}

Finally, the final refocusing arc is developed from three feed positions of $\mathrm{O}_{1}, \mathrm{O}_{2}$ and $\mathrm{O}_{4}$ '. The next step is to specify the feed position for each desired beam. As illustrated in Fig. 5, for the beam radiation at a random angle $\beta$, the related feed $\mathrm{O}_{\mathrm{n}}$ shows the same offset angle as $\beta$, and its focal length is denoted as $L_{\beta}$. With $g=48 \mathrm{~mm}$, $l_{0}=30 \mathrm{~mm}$, and designed maximal radiation angle $\alpha=45^{\circ}$, we can calculate the radius of refocusing arc from (11), as $R=21.8 \mathrm{~mm}$. Then the focal length $L_{\beta}$ can be derived from law of cosines with $R, g-R$ and $\beta$. The feed position can be expressed with $\left(-L_{\beta} \cos \beta,-L_{\beta} \sin \beta\right)$.

As a demonstration here, radiation beams at $0^{\circ}, \pm 10^{\circ}, \pm 20^{\circ}, \pm 30^{\circ}$, $\pm 40^{\circ}, \pm 45^{\circ}$ are given, and the corresponding feed positions are listed in Table I (only positive angles are shown due to structure symmetry).

By shifting the feed horn to different positions according to Table I, different beams are achieved at $72 \mathrm{GHz}$, as shown in Fig. 6. It is noticed that the beam can be scanned to $\pm 43^{\circ}$ with only 3.1-dB drop from the maximum realized gain. The side lobe levels are lower than $-15 \mathrm{~dB}$ for all the beams. For some large beam radiation angles, there is a $1^{\circ}$ or $2^{\circ}$ difference between the 3-D simulated angles and the predefined ones. This can be attributed to the phase errors from the virtual focal length.

In practice, multiple feeds can be used to generate multiple beams, as shown in Fig. 7(a). The model consists of five feed horns for radiations at $0^{\circ}, \pm 20^{\circ}$ and $\pm 43^{\circ}$, respectively. The simulated radiation patterns are given in Fig. 7(b). They are almost the same as the results when a single sliding horn is used as in Fig. 6. Due to the size of the available gain horn in our lab, the angular step of the multiple beams cannot be very small. This could be resolved by using smaller feed horns/waveguides or designing appropriate microstrip antenna arrays.

\section{E. Design Procedure}

According to the theoretical analysis and 3-D simulations discussed before, we summarize the whole design procedure as follows:

Step 1: Choose a proper focal length $l_{0}$ and the required maximal radiation angles $\pm \alpha$;

Step 2: By following (6) and (7), the elliptical contour formula for transmitarray and the specific phase compensation values can be derived;
TABLE I

FEED POSITIONS FOR DIFFERENT BEAM RADIATIONS

\begin{tabular}{|c|c|c|}
\hline \multirow{2}{*}{ Radiation angle $(\boldsymbol{\beta})$} & $\boldsymbol{L}_{\boldsymbol{\beta}} / \mathbf{m m}$ & $\mathbf{O}_{\mathbf{n}}\left(\boldsymbol{x}_{\boldsymbol{n}}, \boldsymbol{y}_{\boldsymbol{n}}\right) /(\mathbf{m m}, \mathbf{m m})$ \\
\hline $0^{\circ}$ & 48 & $(-48,0)$ \\
\hline $10^{\circ}$ & 47.12 & $(-46.4,-8.2)$ \\
\hline $20^{\circ}$ & 44.49 & $(-41.8,-15.2)$ \\
\hline $30^{\circ}$ & 40.11 & $(-34.7,-20)$ \\
\hline $40^{\circ}$ & 33.91 & $(-26,-21.8)$ \\
\hline $45^{\circ}$ & 30 & $(-21.2,-21.2)$ \\
\hline
\end{tabular}

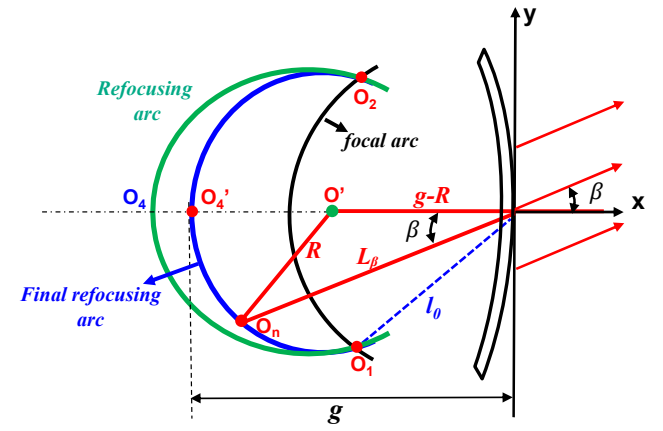

Fig. 5. Schematic for the feed position calculation

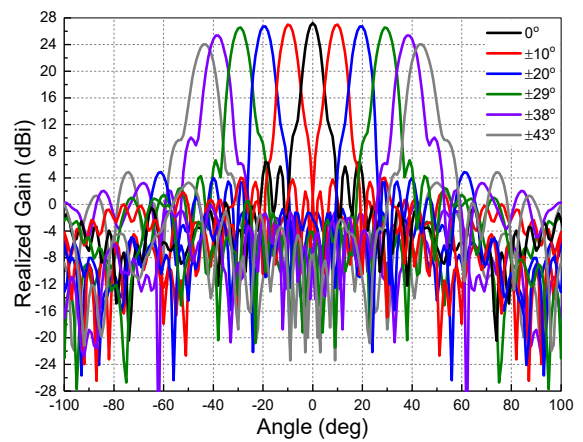

Fig. 6. Simulated multibeam patterns at $72 \mathrm{GHz}$.

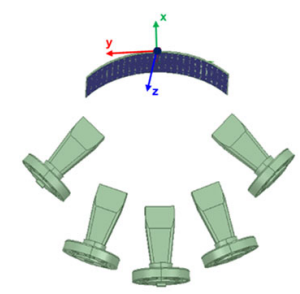

(a)

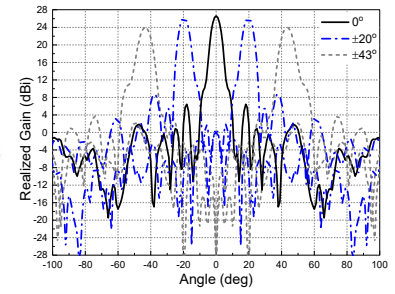

(b)
Fig. 7. (a) The multibeam transmitarray with 5 feed horns for $0^{\circ}, \pm 20^{\circ}$ and $\pm 43^{\circ}$. (b) Simulated radiation patterns with 5 feed horns.

Step 3: Adopt a refocusing design based on (10) to find the feed horn position for boresight radiation and reduce the phase errors of multiple beams on the horizontal plane;

Step 4: Choose the value of $d_{00}$ as the virtual focal length for phase compensation along the vertical straight plane, aiming to balance the radiation performance at $0^{\circ}$ and the maximal radiation angles;

Step 5: Combine phase compensation along the horizontal and vertical planes to decide the transmitarray configuration;

Step 6: Optimize the refocusing position for the boresight radiation to obtain the best $0^{\circ}$ radiation pattern;

Step 7: Draw the final refocusing arc with three known feed positions, and locate different feed horns along this arc to realize multibeam radiations between $-\alpha$ and $\alpha$. 


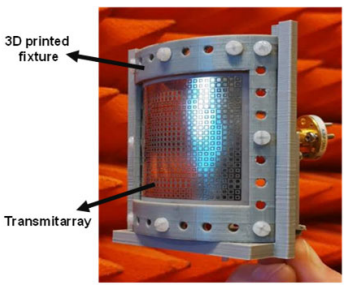

(a)

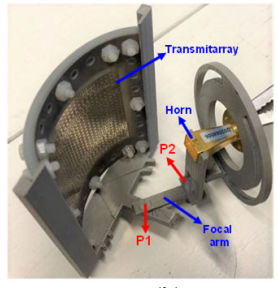

(b)
Fig. 8 Photograph of fabricated prototype. (a) Front view. (b) Side view.

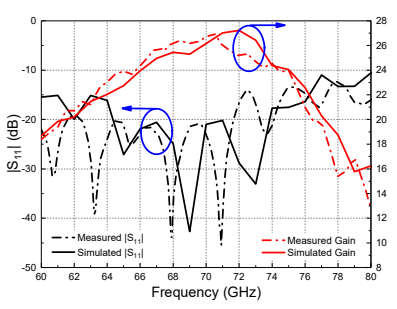

(a)

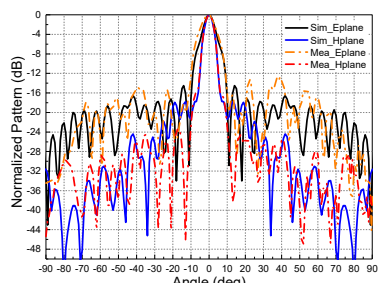

(b)
Fig. 9. Simulated and measured results. (a) $\left|S_{11}\right|$ and realized gain versus frequency for boresight radiation. (b) Boresight radiation patterns at $70.5 \mathrm{GHz}$.

\section{Prototype Fabrication AND MEAsurement}

To verify the developed transmitting aperture for multibeam radiations, an antenna prototype is fabricated and measured. The transmitting aperture consists of two substrate layers with a thickness of $0.254 \mathrm{~mm}$ for each one. The unfolded transmitting surface is fabricated using standard PCB technology. Then the two surfaces are laminated together and attached to a 3-D printed cylindrical frame. Photograph of the transmitarray prototype with the 3-D printed fixture is shown in Fig. 8(a). As seen from Fig. 8(b), P1 and P2 are two screws to connect the focal arm to the array fixture and horn fixture, respectively. $\mathrm{P} 1$ point can be rotated to find different feed positions along the refocusing arc, and P2 point can be rotated to keep the feed horn pointing to the transmitarray centre. Considering the overall cost and available measurement conditions, one feed horn is moved along the refocusing arc for different beams.

Since the transmitarray is a single-port device, the reflection coefficient is measured by connecting the feed horn to one port of the vector network analyzer (VNA). A Keysight VNA N5225A with one $60-90 \mathrm{GHz}$ VNA extender is employed. Simulated and measured $\left|\mathrm{S}_{11}\right|$ for boresight radiation are compared in Fig. 9(a). They are lower than $-10 \mathrm{~dB}$ from $60 \mathrm{GHz}$ to $80 \mathrm{GHz}$. Far-field radiation patterns are measured using a Microwave Vision Group (MVG) compact range antenna measurement system located at University of Technology Sydney, Australia. For boresight radiation, the simulated and measured realized gains versus frequency are also shown in Fig. 9(a). It can be noticed that the operating frequency shifts to a lower band. The simulated peak gain occurs at $72 \mathrm{GHz}$ while the measured one is at $70.5 \mathrm{GHz}$, which corresponds to a shift of $2 \%$. This frequency shift is attributed to multiple reasons, including the phase errors resulting from the fabrication and alignment inaccuracies. Furthermore, a thin PVC layer is employed to laminate the two substrate layers together, which may bring some deviations from the ideal element model in the simulation. The measured $3-\mathrm{dB}$ gain bandwidth is $12.3 \%$ from 65.7 $\mathrm{GHz}$ to $74.3 \mathrm{GHz}$, with a maximal value of $27 \mathrm{dBi}$ at $70.5 \mathrm{GHz}$. The simulated and measured patterns for boresight radiation at $70.5 \mathrm{GHz}$ along E- and H-plane are compared in Fig. 9(b).

The measured multibeam radiation patterns at $66 \mathrm{GHz}, 70.5 \mathrm{GHz}$, and $74 \mathrm{GHz}$ are plotted in Fig. 10(a)-(c), respectively. The realized gains at $70.5 \mathrm{GHz}$ for $0^{\circ}$ and $\pm 43^{\circ}$ are $27 \mathrm{dBi}$ and $24.3 \mathrm{dBi}$, respectively. The peak gain values and sidelobe levels for each beam

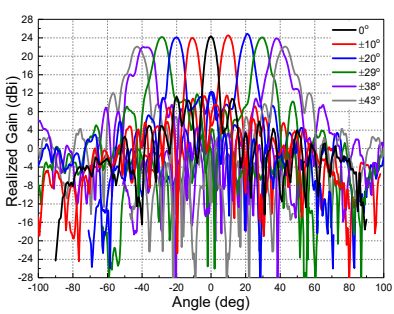

(a)

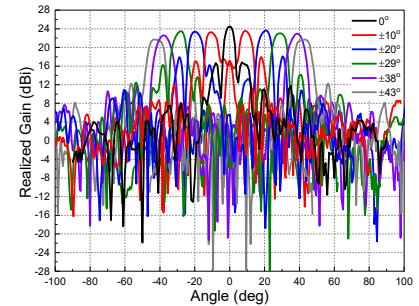

(c)

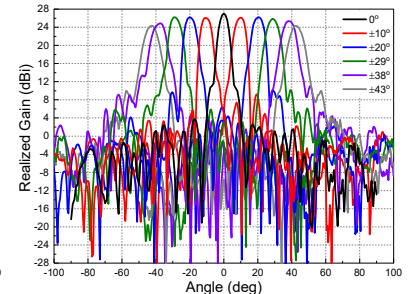

(b)

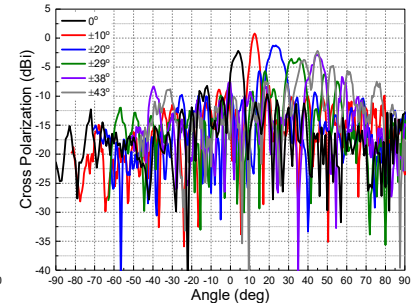

(d)
Fig. 10. Measured multibeam radiation patterns. (a) $66 \mathrm{GHz}$. (b) $70.5 \mathrm{GHz}$. (c) $74 \mathrm{GHz}$. (d) Cross polarization at $70.5 \mathrm{GHz}$.

TABLE II

MEASURED RESULTS OF DIFFERENT SCANNING ANGLES AT 70.5 GHz.

\begin{tabular}{|c|c|c|c|c|c|c|}
\hline $\begin{array}{c}\text { Beam } \\
\text { angle }\end{array}$ & $0^{\circ}$ & $-10^{\circ} /+10^{\circ}$ & $-20^{\circ} /+20^{\circ}$ & $-29^{\circ} / 29^{\circ}$ & $-38^{\circ} /+38^{\circ}$ & $-43^{\circ} /+43^{\circ}$ \\
\hline $\begin{array}{c}\text { Gain } \\
\text { (dBi) }\end{array}$ & 27 & $26 / 26.1$ & $26.2 / 26.2$ & $26.2 / 25.9$ & $25.1 / 25.4$ & $24.3 / 24.3$ \\
\hline $\begin{array}{c}\text { SLL } \\
\text { (dB) }\end{array}$ & -23 & $-19 /-17$ & $-17 /-16$ & $-19 /-16$ & $-16 /-14$ & $-14 /-14$ \\
\hline
\end{tabular}

TABLE III

PERFORMANCE COMPARISON WITH OTHER DESIGNS

\begin{tabular}{|c|c|c|c|c|c|}
\hline $\begin{array}{c}\text { Ref. } \\
\text { No. }\end{array}$ & Frequency & $\begin{array}{c}\text { Maximal beam } \\
\text { coverage }\end{array}$ & $\begin{array}{c}\text { Peak gain } \\
/ \mathbf{d B i}\end{array}$ & $\begin{array}{c}\text { Aperture } \\
\text { efficiency }\end{array}$ & $\begin{array}{c}\text { Scannin } \\
\text { g loss } \\
/ \mathbf{d B}\end{array}$ \\
\hline$[22]$ & $26 \mathrm{GHz}$ & $66^{\circ}\left(-33^{\circ} \sim+33^{\circ}\right)$ & 22.2 & $35.7 \%$ & 1.2 \\
\hline$[23]$ & $28.5 \mathrm{GHz}$ & $60^{\circ}\left(-30^{\circ} \sim+30^{\circ}\right)$ & 25 & $32.3 \%$ & 3.5 \\
\hline$[24]$ & $28 \mathrm{GHz}$ & $54^{\circ}\left(-27^{\circ} \sim+27^{\circ}\right)$ & 24.2 & $24.5 \%$ & 3.7 \\
\hline$[26]$ & $30 \mathrm{GHz}$ & $50^{\circ}\left(0^{\circ} \sim+50^{\circ}\right)$ & 27.3 & $15.1 \%$ & 2.8 \\
\hline$[27]$ & $20 / 30 \mathrm{GHz}$ & $50^{\circ}\left(0^{\circ} \sim+50^{\circ}\right)$ & $24.7 / 27.2$ & $37.3 \% / 29.5 \%$ & $4.4 / 3.7$ \\
\hline$[29]$ & $30 \mathrm{GHz}$ & $\begin{array}{c}82^{\circ}\left(-4^{\circ} \sim+78^{\circ}\right) \\
\text { simulated results }\end{array}$ & 28.9 & $20 \%$ & 3.9 \\
\hline$[30]$ & $25 \mathrm{GHz}$ & $30^{\circ}\left(-15^{\circ} \sim+15^{\circ}\right)$ & 18.7 & $25.1 \%$ & 0.8 \\
\hline $\begin{array}{c}\text { This } \\
\text { work }\end{array}$ & $70.5 \mathrm{GHz}$ & $86^{\circ}\left(-43^{\circ} \sim+43^{\circ}\right)$ & 27 & $34 \%$ & 2.7 \\
\hline
\end{tabular}

angle at $70.5 \mathrm{GHz}$ are listed in Table II. The maximum aperture efficiency is $34 \%$ for the boresight radiation. For other frequencies, the scanning loss is between $2.3 \mathrm{~dB}$ and $2.7 \mathrm{~dB}$. Besides, from Fig. $10(d)$, it is noticeable that the measured cross polarization levels at $70.5 \mathrm{GHz}$ for different radiation angles are all lower than $-20 \mathrm{~dB}$ with respect to the co-polarization level. Similar values are obtained for other frequency points which are not shown here for brevity.

Table III shows the performance comparison between the proposed transmitarray and other reported designs. Regarding the beam coverage, even though a wide range of $82^{\circ}$ is achieved in [29], the aperture efficiency is low and scanning loss is large. The designs from [22] and [30] provide very low scanning losses, but the scanning ranges are limited. It is noticeable that our array has a greater beam scanning range and a small scanning loss without sacrificing the aperture efficiency.

\section{CONCLUSION}

An elliptical cylindrical transmitting aperture is developed for multibeam transmitarrays. The phase compensation and the shape of 
the array are jointly designed to enlarge the beam coverage. A refocusing analysis is introduced to reduce the phase errors of the beams on the horizontal plane. Besides, a virtual focal length is adopted to calculate the phase values on the vertical plane. As a validation, a multibeam prototype at $72 \mathrm{GHz}$ is designed, fabricated and measured, showing good agreement between simulation and measurement. The multibeam radiation angles can cover from $-43^{\circ}$ to $43^{\circ}$ with a 2.7-dB scanning loss. The developed multibeam transmitarrays are expected to find wide applications in $5 \mathrm{G}$ wireless systems and beyond.

\section{REFERENCES}

[1] W. Hong et al. "Multibeam antenna technologies for 5G wireless communications," IEEE Trans. Antennas Propag., vol. 65, no. 12, pp. 6231-6249, Dec. 2017.

[2] X. Huang, Y. J. Guo, J. D. Bunton, "A hybrid adaptive antenna array," IEEE Trans. Wireless Commun., vol. 9, no. 5, pp. 1770-1779, May 2010.

[3] Y. Hu, J. Zhan, Z. H. Jiang, C. Yu, W. Hong, “An orthogonal hybrid analog-digital multibeam antenna array for millimeter-wave massive MIMO systems," IEEE Trans. Antennas Propag., Early access. 2020.

[4] O. Quevedo-Teruel, J. Miao, M. Mattsson, A. Algaba-Brazalez, M. Johansson, L. Manholm, "Glide-symmetric fully metallic Luneburg lens for 5G communications at Ka-band," IEEE Antennas Wireless Propag. Lett., vol. 17, no. 9, pp. 1588-1592, Sep. 2018.

[5] Q. Wu, Z. H. Jiang, O. Quevedo-Teruel, J. P. Turpin, W. Tang, Y. Hao, D. H. Werner, "Transformation optics inspired multibeam lens antennas for broadband directive radiation," IEEE Trans. Antennas Propag., vol. 61, no. 12, pp. 5910-5922, Dec. 2013.

[6] Y. Li, L. Ge, M. Chen, Z. Zhang, Z. Li, J. Wang, "Multibeam 3-D-printed Luneburg lens fed by magnetoelectric dipole antennas for millimeterwave MIMO applications," IEEE Trans. Antennas Propag., vol. 67, no 5, pp. 2923-2933, May 2019.

[7] Z. H. Jiang, F. Wu, X.-W. Zhu, Q. Ren, P. L. Werner, D. H. Werner, "Metasurface-based circularly-polarized multibeam reflect-/transmitarrays," 14th European Conference on Antennas and Propagation (EuCAP), 2020.

[8] A. H. Abdelrahman, P. Nayeri, A. Z. Elsherbeni, and F. Yang, "Singlefeed quad-beam transmitarray antenna design," IEEE Trans. Antennas Propag., vol. 64, no. 3, pp. 953-959, Mar. 2016.

[9] A. Aziz, F. Yang, S. Xu, M. Li, "A low-profile quad-beam transmitarray,' IEEE Antennas Wireless Propag. Lett., vol. 19, no. 8, pp. 1340-1344, Aug. 2020

[10] P. Nayeri, F. Yang, and A. Z. Elsherbeni, "Design of single-feed reflectarray antennas with asymmetric multiple beams using the particle swarm optimization method," IEEE Trans. Antennas Propag., vol. 61, no. 9, pp. 4598-4605, Sep. 2013.

[11] Z. H. Jiang, Y, Zhang, W, Hong, "Anisotropic impedance surfaceenabled low-profile broadband dual-circularly polarized multibeam reflectarrays for Ka-band applications," IEEE Trans. Antennas Propag., vol. 68 , no. 8, pp. 6441-6446, Aug. 2020.

[12] Y. Hu, W. Hong, Z. H. Jiang, "A multibeam folded reflectarray antenna with wide coverage and integrated primary sources for millimeter-wave massive MIMO applications," IEEE Trans. Antennas Propag., vol. 66, no. 12 , pp. 6875-6882, Dec. 2018.

[13] M. Ansari, H. Zhu, N. Shariati, Y. J. Guo, "Compact planar beamforming array with endfire radiating elements for $5 \mathrm{G}$ applications," IEEE Trans. Antennas Propag., vol. 67, no. 11, pp. 6859-6869, Nov. 2019.

[14] J.-W. Lian, Y.-L. Ban, C. Xiao, Z.-F. Yu, "Compact substrate-integrated $4 \times 8$ Butler matrix with sidelobe suppression for millimeter-wave multibeam application," IEEE Antennas Wireless Propag. Lett., vol. 17, no. 5, pp. 928-932, May 2018.

[15] J. Y. Lau, S. V. Hum, "Reconfigurable transmitarray design approaches for beamforming applications," IEEE Trans. Antennas Propag., vol. 60, no. 12 , pp. 5679-5689, Dec. 2012.

[16] L. D. Palma, A. Clemente, L. Dussopt, R. Sauleau, P. Potier, and P. Pouliguen, "Circularly polarized transmitarray with sequential rotation in Ka-band," IEEE Trans. Antennas Propag., vol. 63, no. 11, pp. 5118-5124, Nov. 2015
[17] A. H. Abdelrahman, A. Z. Elsherbeni, F. Yang, "High-gain and broadband transmitarray antenna using triple-layer spiral dipole elements," IEEE Antennas Wireless Propag. Lett., vol. 13, pp. 1288-1291, 2014.

[18] Q. Luo, S. Gao, M. Sobhy, X. Yang, "Wideband transmitarray with reduced profile," IEEE Antennas Wireless Propag. Lett., vol. 17, no. 3, pp. 450-453, Mar. 2018.

[19] C. Pfeiffer and A. Grbic, "Millimeter-wave transmitarrays for wavefront and polarization control," IEEE Trans. Microw. Theory Tech., vol. 61, no. 12, pp. 4407-4417, Dec. 2013.

[20] M. Frank, F. Lurz, R. Weigel and A. Koelpin, "Low-cost transmitarray antenna designs with $\pm 70^{\circ}$ beam steering range in V-band," 2019 12th German Microwave Conference (GeMiC), Stuttgart, Germany, 2019.

[21] P. Nayeri, F. Yang, A. Z. Elsherbeni, "Bifocal design and aperture phase optimizations of reflectarray antennas for wide-angle beam scanning performance," IEEE Trans. Antennas Propag., vol. 61, no. 9, pp. 45884597, Sep. 2013.

[22] Z. H. Jiang, Y. Zhang, J. Xu, Y. Yu, W. Hong, "Integrated broadband circularly polarized multibeam antennas using Berry-phase transmitarrays for Ka-band applications," IEEE Trans. Antennas Propag. vol. 68 , no. 2 , pp. $859-872$, Feb. 2020.

[23] G. Liu, M. R. D. Kodnoeih, T. K. Pham, E. M. Cruz, D. GonzálezOvejero, R. Sauleau, "A millimeter-wave multibeam transparent transmitarray antenna at Ka-band," IEEE Antennas Wireless Propag. Lett., vol. 18, no. 4, pp. 631-635, Apr. 2019.

[24] M. Jiang, Z. N. Chen, Y. Zhang, W. Hong, X. Xuan, "Metamaterial-based thin planar lens antenna for spatial beamforming and multibeam massive MIMO," IEEE Trans. Antennas Propag., vol. 65, no. 2, pp. 464-472, Feb. 2017.

[25] G. Li, Z. Wang, Y. Ge, "Multibeam folded transmitarray antenna for massive MIMO applications," International Workshop on Electromagnetics: Applications and Student Innovation Competition (iWEM), Sep. 2019.

[26] E. B. Lima, S. A. Matos, J. R. Costa, C. A. Fernandes, N. J. G. Fonseca, "Circular polarization wide-angle beam steering at Ka-band by in-plane translation of a plate lens antenna," IEEE Trans. Antennas Propag., vol. 63, no. 12, pp. 5443-5455, Dec. 2015.

[27] S. A. Matos, E. B. Lima, J. S. Silva, J. R. Costa, C. A. Fernandes, N. J. G. Fonseca, J. R. Mosig, "High gain dual-band beam-steering transmit array for satcom terminals at Ka-band," IEEE Trans. Antennas Propag. vol. 65, no. 7, pp. 3528-3539, Jul. 2017.

[28] Y. Hou, L. Chang, Y. Li, Z. Zhang, Z. Feng, "Linear multibeam transmitarray based on the sliding aperture technique," IEEE Trans. Antennas Propag., vol. 66, no. 8, pp. 3948-3958, Aug. 2018.

[29] A. Arraianol, et al. "Ultra-wide beam scanning using a conformal transmit-array for Ka-band." the13th European Conference on Antennas and Propagation (EuCAP), IEEE, 2019.

[30] P.-Y. Qin, L.-Z. Song, Y. J. Guo, "Beam steering conformal transmitarray employing ultra-thin triple-layer slot elements," IEEE Trans. Antennas Propag., vol. 67, no. 8, pp. 5390-5398, Aug. 2019.

[31] J. Ruze, "Wide-angle metal-plate optics," Proc. IRE, vol. 38, no. 1, pp. 53-69, Jan. 1950

[32] L.-Z. Song, P.-Y. Qin, Y. J. Guo, "A high-efficiency conformal transmitarray antenna employing dual-layer ultra-thin Huygens element," IEEE Trans. Antennas Propag., IEEE Trans. Antennas Propag., vol. 69, no. 2 , pp. 848-858, Feb. 2021.

[33] https://www.ainfoinc.com/amfilerating/file/download/file_id/1229/. 